Supplement of Hydrol. Earth Syst. Sci., 23, 3553-3570, 2019

https://doi.org/10.5194/hess-23-3553-2019-supplement

(C) Author(s) 2019. This work is distributed under

the Creative Commons Attribution 4.0 License.

(c) (1)

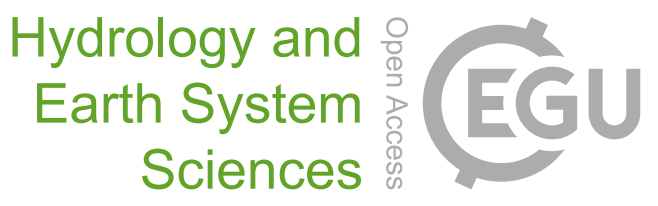

Supplement of

\title{
Partitioning snowmelt and rainfall in the critical zone: effects of climate type and soil properties
}

John C. Hammond et al.

Correspondence to: John C. Hammond (john.christopher.hammond@gmail.com)

The copyright of individual parts of the supplement might differ from the CC BY 4.0 License. 
Model Calibration

\section{Supplementary material}

We developed simulations using two rounds of historical climate inputs, the first as a spin up period, and the second for calibration. Calibration consisted of adjusting the hydraulic conductivity of the bottom layer, which controlled how much water was retained in the soil profile. Rather than force-fitting, our goal was to produce simulations with similar timing of wetting and drying to observations. This approach is consistent with other studies using HYDRUS - 1D, which also started with basic soils data and application of the ROSETTA pedotransfer function (i.e. Scott et al., 2000) and then calibrated to observed water content measurements by adjusting permeability of the "bedrock" layer (Flint et al., 2008). We evaluated performance using mean bias and the Nash Sutcliffe model coefficient of efficiency (NSCE). We tested the simulation configuration by comparing to observed volumetric water content (VWC) at 5, 20 and $50 \mathrm{~cm}$ depths for the three selected soil profile sites (Figure S1, Table S1). Figure S1 shows observed and simulated time series of VWC at 5, 20 and $50 \mathrm{~cm}$ depth after completed calibration where the bedrock $K_{s}$ for each site was set to $1 / 10$ that of the later above.

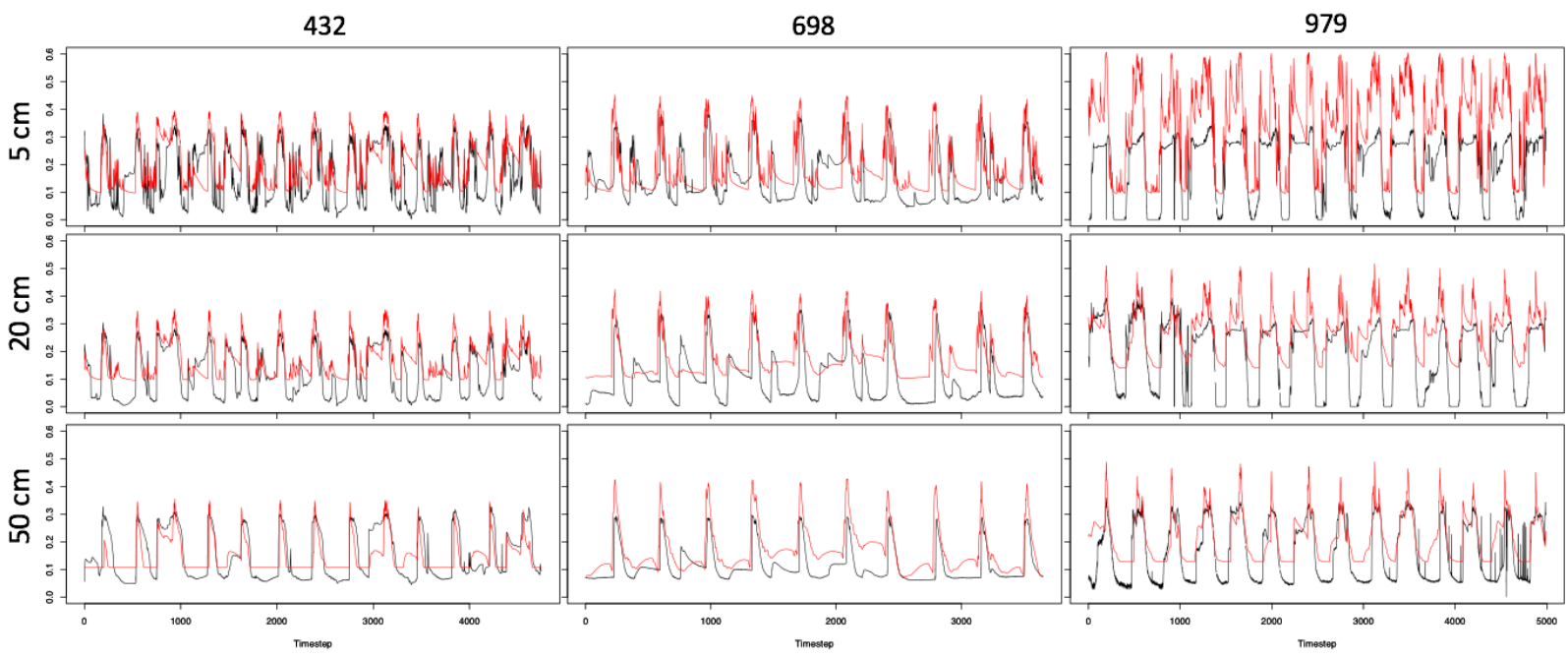

Figure S1. Observed (black) and simulated (red) time series of volumetric water content at 5, 20 and $50 \mathrm{~cm}$ for SNOTEL sites and profiles 432 Currant Creek, 698 Pole Creek R.S., and 979 Van Wyck. 
Table S1. Calibration metrics Nash-Sutcliffe model efficiency coefficient (NSCE) and mean bias for VWC at 5,20 and $50 \mathrm{~cm}$ depth.

\begin{tabular}{lrrr}
\hline Metric & SNOTEL432 & SNOTEL 698 & SNOTEL 979 \\
\hline NSCE $5 \mathrm{~cm}$ & -0.33 & 0.15 & -0.82 \\
NSCE $20 \mathrm{~cm}$ & 0.45 & 0.17 & 0.22 \\
NSCE $50 \mathrm{~cm}$ & 0.58 & 0.14 & 0.48 \\
Mean bias $5 \mathrm{~cm}$ & -6.7 & 9.4 & 46.9 \\
Mean bias $20 \mathrm{~cm}$ & 8.5 & 10.4 & 22.4 \\
Mean bias $50 \mathrm{~cm}$ & -7.4 & 10 & 19.7
\end{tabular}

Because the purpose of this study was to use the modeling framework to examine how the water balance is affected by changes in snow and soil properties, the simulations are not intended to replicate site-specific conditions exactly. Better representation of site-specific conditions would require finer spatial and temporal scale measurements of surface boundary conditions (input, potential evapotranspiration); information on root distribution, root water uptake, and hydraulic properties of subsurface layers below the soil profile. Lateral redistribution of water and preferential flow may also affect soil moisture at these sites, but these processes are not represented in the simulations. Because of the points above, several additional analyses were performed in order to understand the effects of our model parameterization on our reported results.

To examine the sensitivity of our simulation results to the input data time step, we modeled several SNOTEL sites (531 Hoosier Pass, CO; 335 Berthoud Summit, CO; and 1123 Longdraw Reservoir, CO) at both hourly and daily time steps using hourly snowmelt and rainfall data compiled in a previous study (Webb et al., 2017). Results from these simulations showed overall similar response between daily and hourly surface runoff and deep drainage with slightly greater cumulative surface runoff from hourly simulations (hourly $103-124 \%$ of daily simulations) and slightly greater cumulative deep drainage (hourly $88-96 \%$ of daily simulations) from daily simulations (Figure S3, Table S5).

We also investigated the mechanism by which surface runoff was being generated in simulations and the timing of deep drainage generation relative to saturated conditions within the soil. To determine the mechanism for generating $\mathrm{Q}$ we used time series of $5 \mathrm{~cm}$ VWC with a set of rules 
for identifying runoff generation mechanism. Any time step with overland flow and saturation $>0.95$ was considered saturation excess, whereas time steps with overland flow and saturation $\leq 0.95$ were considered infiltration excess. For all sites together, the mean fraction of annual runoff from saturation excess is $97 \%$ (Figure S4). For wet and dry sites combined, $57 \%$ of annual deep drainage occurred when the 5, 20,50 and $100 \mathrm{~cm}$ observation nodes were saturated (ex. Figure S5). At wet sites $60 \%$ of deep drainage occurred during these periods of saturation as compared to $50 \%$ at dry sites. Weak positive relationships $(\mathrm{r}<0.25)$ exist between $\mathrm{P}$ and peak SWE and the fraction of deep drainage occurring when all observation nodes were saturated.

Finally, we examined the fraction of input occurring as mixed input (rain on snow) to understand how our selection of binary rainfall and snowmelt events for event scale analysis compares to the distribution of inputs across all sites and all years (Table S6).

Table S2. Cumulative deep drainage and surface runoff for snowmelt and rain periods compared between hourly and daily simulations.

\begin{tabular}{llrrr}
\hline Variable & Cumulative comparison & Hoosier & Berthoud & Longdraw \\
\hline Deep drainage & Rain hourly percent of Daily & 96 & 95 & 88 \\
Deep drainage & Snow hourly percent of Daily & 89 & 91 & 96 \\
Runoff & Rain hourly percent of Daily & 108 & 124 & 112 \\
Runoff & Snow hourly percent of Daily & 108 & 104 & 103
\end{tabular}



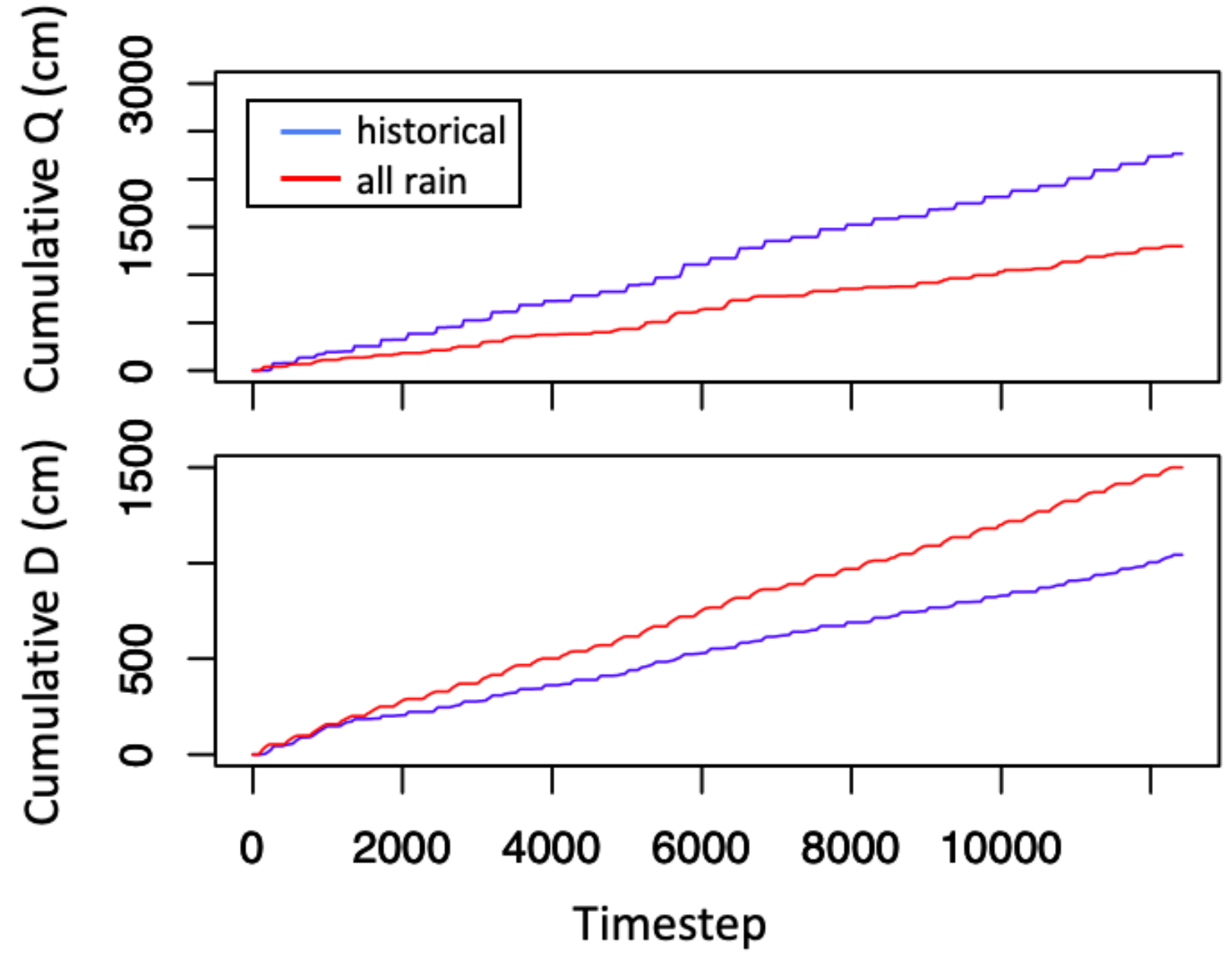

Figure S2. Daily cumulative surface runoff (Q) and deep drainage (D) for historical (blue) and all rain (red) scenarios at SNOTEL site 418 Divide, MT using loam profile from SNOTEL site 1056. Here, lower cumulative deep drainage from the historical scenario likely results from greater input being partitioned into surface runoff. 

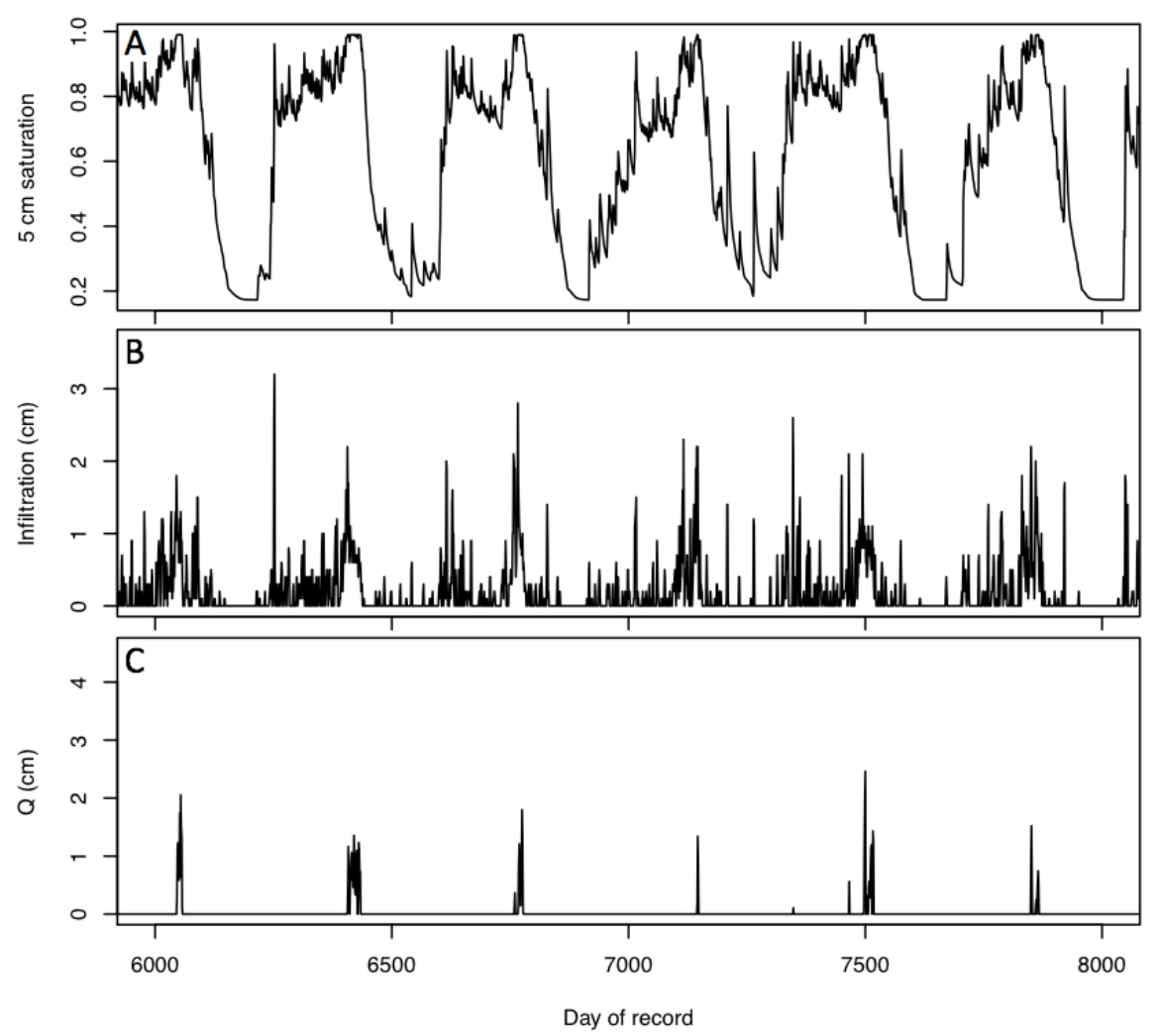

Figure S3. Example daily time series of $5 \mathrm{~cm}$ saturation (A), infiltration (B) and runoff (C) for SNOTEL site 352 historical scenario. 

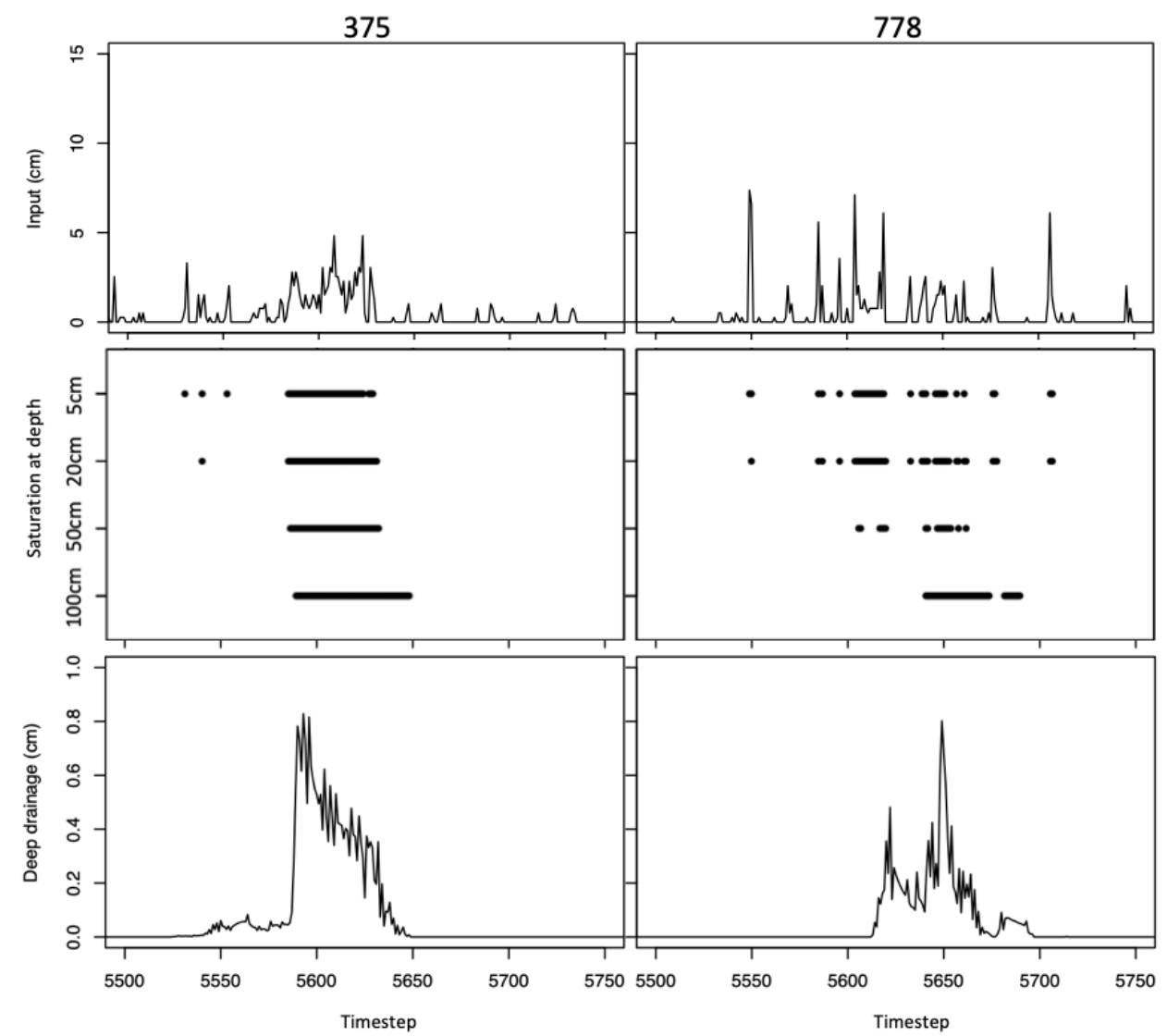

Figure S4. Daily series of input, saturation at 5,20,50, and $100 \mathrm{~cm}$ depths and deep drainage for two sites SNOTEL 375 Bumping Ridge, WA and SNOTEL 778 Spratt Creek, CA using loam profile from SNOTEL site 1056.

$\underline{\text { Relationships between partitioned components and explanatory variables }}$

Below we provide correlations between explanatory climatic variables and partitioned response and precipitation normalized response variables (Table S2). Tables S3 and S4 then show correlations and slopes of relationships between precipitation normalized response variables (Q/P, D/P, ET/P) and explanatory variables (snow fraction, annual average saturation at $100 \mathrm{~cm}$, and input concentration index). Figure S2 displays the relationship between transpiration and precipitation colored by the ratio of evapotranspiration to precipitation (ET/P). We include this figure as a way of displaying the conditions under which ET/P exceeds 1. 
Table S3. Correlations between annual values of climatic and water balance terms for historical input scenarios. Variables included are precipitation $(\mathrm{P})$, potential evapotranspiration (PET), peak snow water equivalent (peak SWE), snow persistence (SP), input concentration index (ICI), snowmelt fraction of input (sfrac), mean saturation at $100 \mathrm{~cm}$ depth (Sat100) and other depths, surface runoff $(\mathrm{Q})$, deep drainage $(\mathrm{D})$, transpiration $(\mathrm{T})$, evaporation $(\mathrm{E})$, and aridity index (P/PET). P-value of correlation, $*<0.5$, $* *<0.01$, $* * *<0.001$.

\begin{tabular}{|c|c|c|c|c|c|c|c|c|c|c|}
\hline & $\mathrm{D}$ & ICI & $\mathrm{P}$ & PET & SWE & sfrac & Sat5 & Sat20 & Sat50 & Sat100 \\
\hline ICI & $0.44 * * *$ & & & & & & & & & \\
\hline $\mathrm{P}$ & $0.92 * * *$ & $0.84 * * *$ & & & & & & & & \\
\hline PET & $-0.55 * * *$ & $-0.22 * * *$ & $-0.43 * * *$ & & & & & & & \\
\hline SWE & $0.78 * * *$ & $0.69 * * *$ & $0.74 * * *$ & $-0.46 * * *$ & & & & & & \\
\hline sfrac & $0.31 * * *$ & $0.29 * * *$ & $-0.15 * *$ & $-0.20 * *$ & $0.55 * * *$ & & & & & \\
\hline Sat5 & $0.84 * * *$ & $0.57 * * *$ & $0.74 * * *$ & $-0.77 * * *$ & $0.70 * * *$ & $0.36 * * *$ & & & & \\
\hline Sat20 & $0.86 * * *$ & $0.60 * * *$ & $0.75 * * *$ & $-0.73 * * *$ & $0.72 * * *$ & $0.37 * * *$ & $0.99 * * *$ & & & \\
\hline Sat50 & $0.88 * * *$ & $0.64 * * *$ & $0.76 * * *$ & $-0.65 * * *$ & $0.73 * * *$ & $0.36 * * *$ & $0.95 * * *$ & 0.97 & & \\
\hline Sat100 & $0.86 * * *$ & $0.63 * * *$ & $0.73 * * *$ & $-0.55 * * *$ & $0.70 * * *$ & $0.34 * * *$ & $0.84 * * *$ & $0.88 * * *$ & $0.93 * * *$ & \\
\hline Q & $0.91 * * *$ & $0.85 * * *$ & $0.97 * * *$ & $-0.42 * * *$ & $0.83 * * *$ & $0.30 * * *$ & $0.73 * * *$ & $0.75 * * *$ & $0.76^{* * *}$ & $0.73 * * *$ \\
\hline ET & -0.04 & $0.16 * *$ & 0.08 & 0.06 & 0.04 & $0.27 * * *$ & $0.21 * * *$ & $0.22 * * *$ & $0.17 * * *$ & $0.15 * * *$ \\
\hline $\mathrm{D} / \mathrm{P}$ & $0.77 * * *$ & $0.45 * * *$ & $0.51 * * *$ & $-0.52 * * *$ & $0.56^{* * *}$ & $0.20 * * *$ & $0.73 * * *$ & $0.77 * * *$ & $0.83 * * *$ & $0.86 * * *$ \\
\hline P/PET & $0.93 * * *$ & $0.79 * * *$ & $0.98 * * *$ & $-0.55 * * *$ & $0.76 * * *$ & -0.09 & $0.79 * * *$ & $0.79 * * *$ & $0.79 * * *$ & $0.74 * * *$ \\
\hline $\mathrm{Q} / \mathrm{P}$ & $0.81 * * *$ & $0.80 * * *$ & $0.76 * * *$ & $-0.38 * * *$ & $0.86 * * *$ & $0.41 * * *$ & $0.72 * * *$ & $0.76 * * *$ & $0.80 * * *$ & $0.80 * * *$ \\
\hline ET/P & $-0.87 * * *$ & $-0.76 * * *$ & $-0.83 * * *$ & $0.44 * * *$ & $-0.76 * * *$ & -0.02 & $-0.71 * * *$ & $-0.75 * * *$ & $-0.81 * * *$ & $-0.82 * * *$ \\
\hline
\end{tabular}


Table S3 continued. Correlations between annual values of climatic and water balance terms for historical input scenarios. Variables included are precipitation (P), potential evapotranspiration (PET), peak snow water equivalent (peak SWE), snow persistence (SP), average melt rate over the year (melt rate), mean saturation at $100 \mathrm{~cm}$ depth (Sat 100) and other depths, surface runoff (Q), deep drainage (D), transpiration (T), evaporation (E), and aridity index (P/PET). P-value of correlation, $*<0.5, * *<0.01, * * *<0.001$.

\begin{tabular}{llllll}
\hline & $\mathrm{Q}$ & $\mathrm{ET}$ & $\mathrm{D} / \mathrm{P}$ & $\mathrm{P} / \mathrm{PET}$ & $\mathrm{Q} / \mathrm{P}$ \\
\hline $\mathrm{Q}$ & & & & & \\
$\mathrm{ET}$ & 0.04 & & & & \\
$\mathrm{D} / \mathrm{P}$ & $0.52 * * *$ & -0.01 & & & \\
$\mathrm{P} / \mathrm{PET}$ & $0.96 * * *$ & -0.01 & $0.54 * * *$ & & \\
$\mathrm{Q} / \mathrm{P}$ & $0.84 * * *$ & $0.14 * *$ & $0.70^{* * *}$ & $0.73 * * *$ & \\
$\mathrm{ET} / \mathrm{P}$ & $-0.80 * * *$ & 0.04 & $-0.75 * * *$ & $-0.81 * * *$ & $-0.84 * * *$
\end{tabular}

Table S4. Correlation coefficients, slopes of linear fit between explanatory and response variable, and whether slopes for different climate types are significantly different for the ratio of Q, D and ET to P vs snowmelt fraction of input, mean saturation at $100 \mathrm{~cm}$, and input concertation index (ICI). Whether slopes were significantly different was estimated by the p-value of a pairwise difference in linear regression slope test where $*<0.5, * *<0.01, * * *<0.001$. Results from the climate scenarios in Figure 5, loam soil type. Dry P/PET $<1=$, Wet P/PET $>1$.

\begin{tabular}{|c|c|c|c|c|c|c|c|c|}
\hline \multirow[b]{2}{*}{ Variables } & \multicolumn{3}{|l|}{ All } & \multicolumn{2}{|l|}{ Dry } & \multicolumn{2}{|l|}{ Wet } & \multirow[b]{2}{*}{ Slopes different? } \\
\hline & $\mathrm{r}$ & & Slope & $\mathrm{r}$ & Slope & $\mathrm{r}$ & Slope & \\
\hline $\mathrm{Q} / \mathrm{P}$ vs snowmelt fraction & 0.41 & $* * *$ & 0.53 & 0.44 & $* * * 0.41$ & $0.41 * * *$ & 0.38 & $* * *$ \\
\hline $\mathrm{D} / \mathrm{P}$ vs snowmelt fraction & 0.20 & $* * *$ & 0.07 & 0.32 & $* * * 0.10$ & -0.02 & 0.00 & no \\
\hline $\mathrm{ET} / \mathrm{P}$ vs snowmelt fraction & -0.02 & & 0.05 & 0.05 & 0.07 & $0.30 * * *$ & 0.33 & $*$ \\
\hline $\mathrm{Q} / \mathrm{P}$ vs Sat 100 & 0.77 & $* * *$ & 0.87 & 0.60 & $* * * 0.59$ & $0.52 * * *$ & 0.65 & $*$ \\
\hline D/P vs Sat 100 & 0.85 & $* * *$ & 0.27 & 0.78 & $* * * 0.27$ & $0.65 * * *$ & 0.22 & no \\
\hline ET/P vs Sat 100 & -0.79 & $* * *$ & 1.41 & -0.41 & $* * * 0.62$ & $-0.62 * * *$ & 0.92 & $* *$ \\
\hline $\mathrm{Q} / \mathrm{P}$ vs ICI & 0.80 & $* * *$ & 0.151 & 0.76 & $* * * 0.177$ & $0.66 * * *$ & 0.094 & $*$ \\
\hline D/P vs ICI & 0.43 & $* * *$ & 0.57 & 0.49 & $* * * 0.041$ & $-0.15 *$ & 0.006 & no \\
\hline ET/P vs ICI & -0.75 & $* * *$ & -0.230 & -0.61 & $* * *-0.219$ & $-0.62 * * *$ & -0.107 & no \\
\hline
\end{tabular}


Table S5. Correlation coefficients, slopes of linear fit between explanatory and response variable, and whether slopes for different soil textures are significantly different for the ratio of Q, D and ET to P vs snowmelt fraction of input, mean saturation at $100 \mathrm{~cm}($ Sat 100$)$, and input concertation index (ICI). Whether slopes were significantly different was estimated by the p-value of a pairwise difference in linear regression slope test where $*<0.5, * *<0.01, * * *<0.001$.

\begin{tabular}{|c|c|c|c|c|c|c|c|}
\hline \multirow[b]{2}{*}{ Variables } & \multicolumn{2}{|c|}{ Sandy loam } & \multicolumn{2}{|c|}{ Sandy clay loam } & \multicolumn{2}{|c|}{ Loam } & \multirow[b]{2}{*}{ Slopes different? } \\
\hline & $\mathrm{r}$ & Slope & $\mathrm{r}$ & Slope & $\mathrm{r}$ & Slope & \\
\hline $\mathrm{Q} / \mathrm{P}$ vs snowmelt fraction & 0.42 & $* * * 0.53$ & 0.39 & $* * * 0.52$ & 0.38 & $* * * 0.50$ & no \\
\hline $\mathrm{D} / \mathrm{P}$ vs snowmelt fraction & 0.28 & $* * * 0.10$ & 0.24 & $* * * 0.09$ & 0.21 & $* * * 0.08$ & no \\
\hline ET/P vs snowmelt fraction & 0.01 & 0.01 & 0.00 & 0.01 & 0.01 & 0.01 & no \\
\hline $\mathrm{Q} / \mathrm{P}$ vs Sat100 & 0.75 & $* * * 0.78$ & 0.77 & $* * * 0.93$ & 0.80 & $* * * 0.86$ & $* * *$ \\
\hline D/P vs Sat100 & 0.76 & $* * * 0.22$ & 0.81 & $* * * 0.27$ & 0.85 & $* * * 0.27$ & $*$ \\
\hline ET/P vs Sat 100 & -0.81 & $* * * 1.34$ & -0.79 & $* * * 1.54$ & -0.80 & $* * * 1.43$ & $* *$ \\
\hline $\mathrm{Q} / \mathrm{P}$ vs ICI & 0.67 & $* * * 0.093$ & 0.66 & $* * * 0.096$ & 0.66 & $* * * 0.097$ & no \\
\hline D/P vs ICI & 0.23 & $* * * 0.009$ & 0.29 & $* * * 0.012$ & 0.32 & $* * * 0.050$ & no \\
\hline ET/P vs ICI & -0.63 & $* * *-0.136$ & -0.61 & $* * *-0.144$ & -0.61 & $* * *-0.148$ & no \\
\hline
\end{tabular}




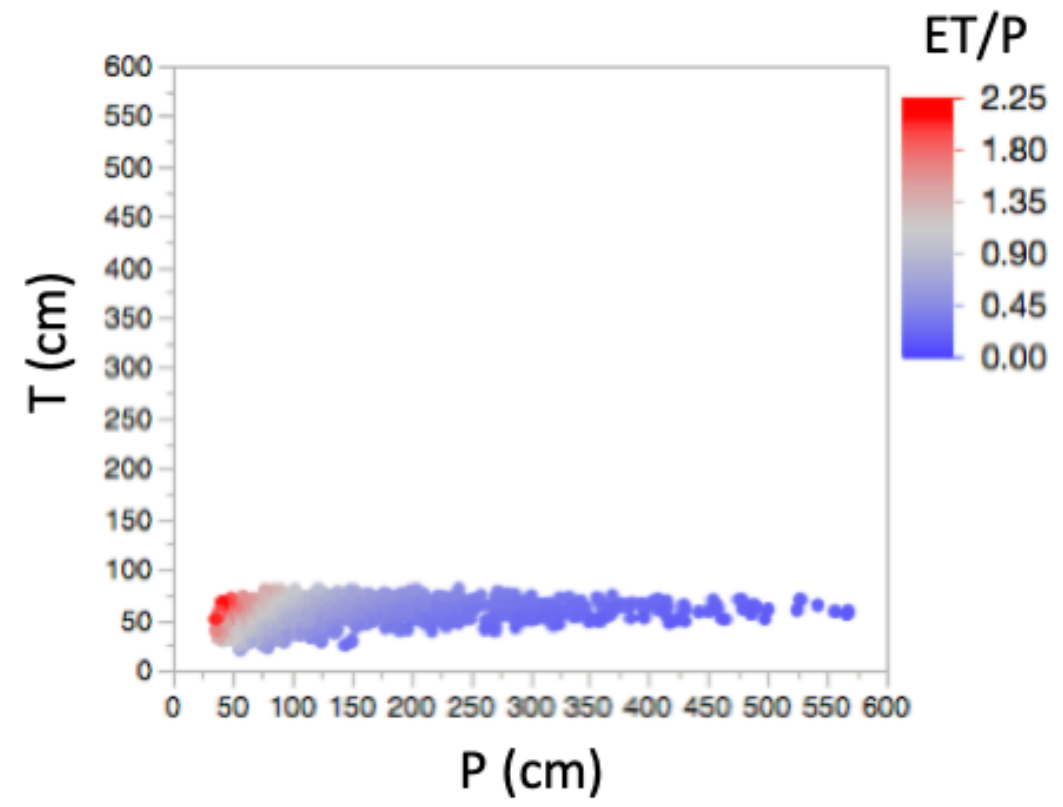

Figure S5. Historical annual transpiration vs precipitation colored by the ratio of annual evapotranspiration to precipitation for all sites on SNOTEL 1056 loam profile.

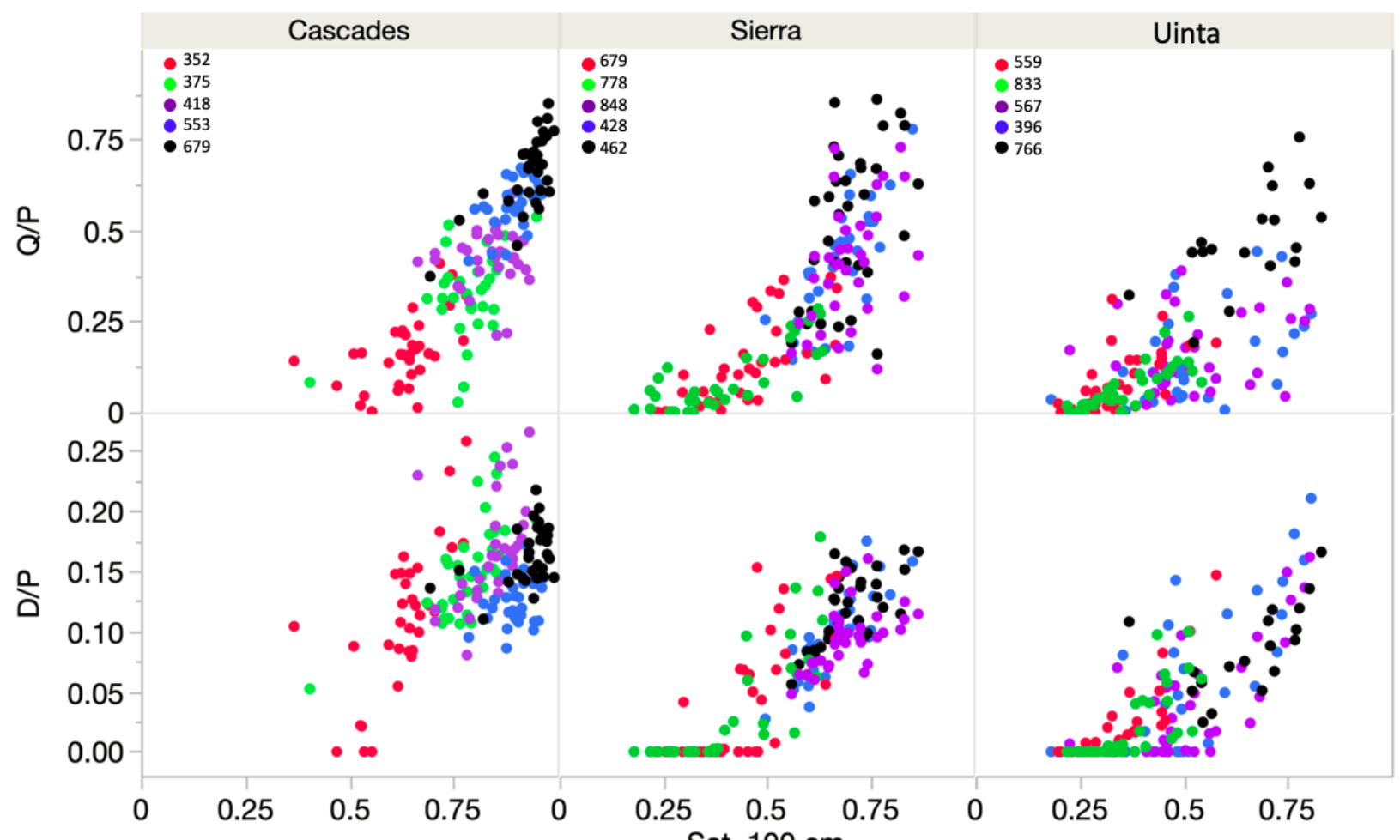

Sat. $100 \mathrm{~cm}$

Figure S6. Annual Q/P and D/P vs annual mean saturation at $100 \mathrm{~cm}$ depth by region. 


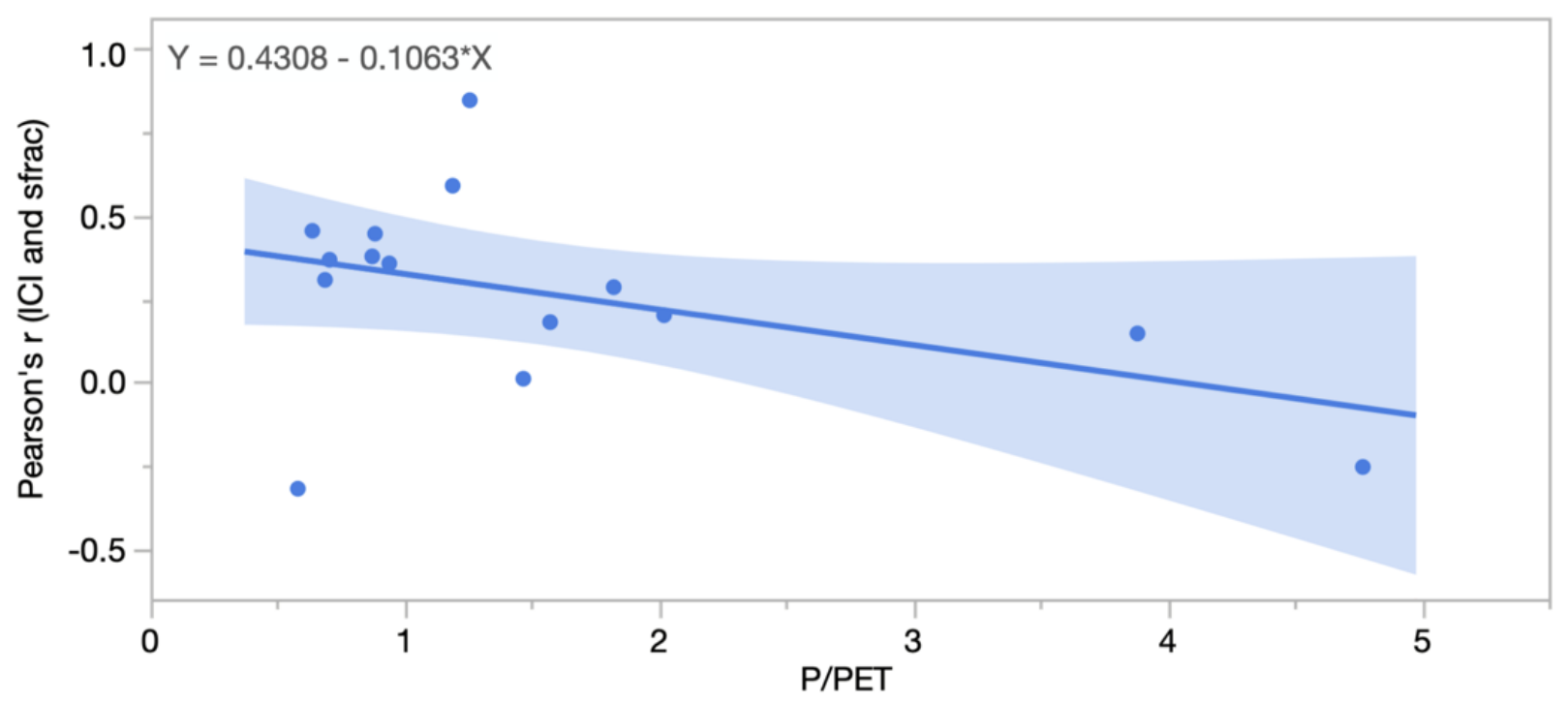

Figure S7. Linear relationship between Pearson's correlation coefficient of ICI and snowmelt fraction of input vs each site's mean annual aridity index. Pearson's correlation coefficient of ICI and snowmelt fraction relationship vs mean annual aridity index is -0.44 .

Table S6. Fraction of input from rainfall, mixed input and snowmelt by site and across all sites.

\begin{tabular}{rrrr}
\hline Site & Rain fraction & Rain on snow fraction & Snowmelt fraction \\
\hline 352 & 0.23 & 0.45 & 0.32 \\
375 & 0.18 & 0.37 & 0.45 \\
396 & 0.39 & 0.52 & 0.10 \\
418 & 0.12 & 0.45 & 0.43 \\
428 & 0.15 & 0.51 & 0.34 \\
462 & 0.11 & 0.67 & 0.22 \\
481 & 0.32 & 0.52 & 0.16 \\
553 & 0.25 & 0.20 & 0.54 \\
559 & 0.45 & 0.43 & 0.12 \\
679 & 0.11 & 0.54 & 0.35 \\
697 & 0.21 & 0.54 & 0.25 \\
766 & 0.18 & 0.70 & 0.12 \\
778 & 0.46 & 0.27 & 0.27 \\
833 & 0.42 & 0.45 & 0.13 \\
848 & 0.15 & 0.48 & 0.38 \\
All sites & $\mathbf{0 . 2 5}$ & $\mathbf{0 . 4 7}$ & $\mathbf{0 . 2 8}$
\end{tabular}

